\title{
Chapter 13 \\ Case Studies of Capacity Building for Biodiversity Monitoring
}

\author{
Dirk S. Schmeller, Christos Arvanitidis, Monika Böhm, \\ Neil Brummitt, Eva Chatzinikolaou, Mark J. Costello, Hui Ding, \\ Michael J. Gill, Peter Haase, Romain Julliard, Jaime García-Moreno, \\ Nathalie Pettorelli, Cui Peng, Corinna Riginos, Ute Schmiedel, \\ John P. Simaika, Carly Waterman, Jun Wu, Haigen Xu \\ and Jayne Belnap
}

\begin{abstract}
Monitoring the status and trends of species is critical to their conservation and management. However, the current state of biodiversity monitoring is insufficient to detect such for most species and habitats, other than in a few localised areas. One of the biggest obstacles to adequate monitoring is the lack of local capacity to carry out such programs. Thus, building the capacity to do such monitoring is imperative. We here highlight different biodiversity monitoring
\end{abstract}

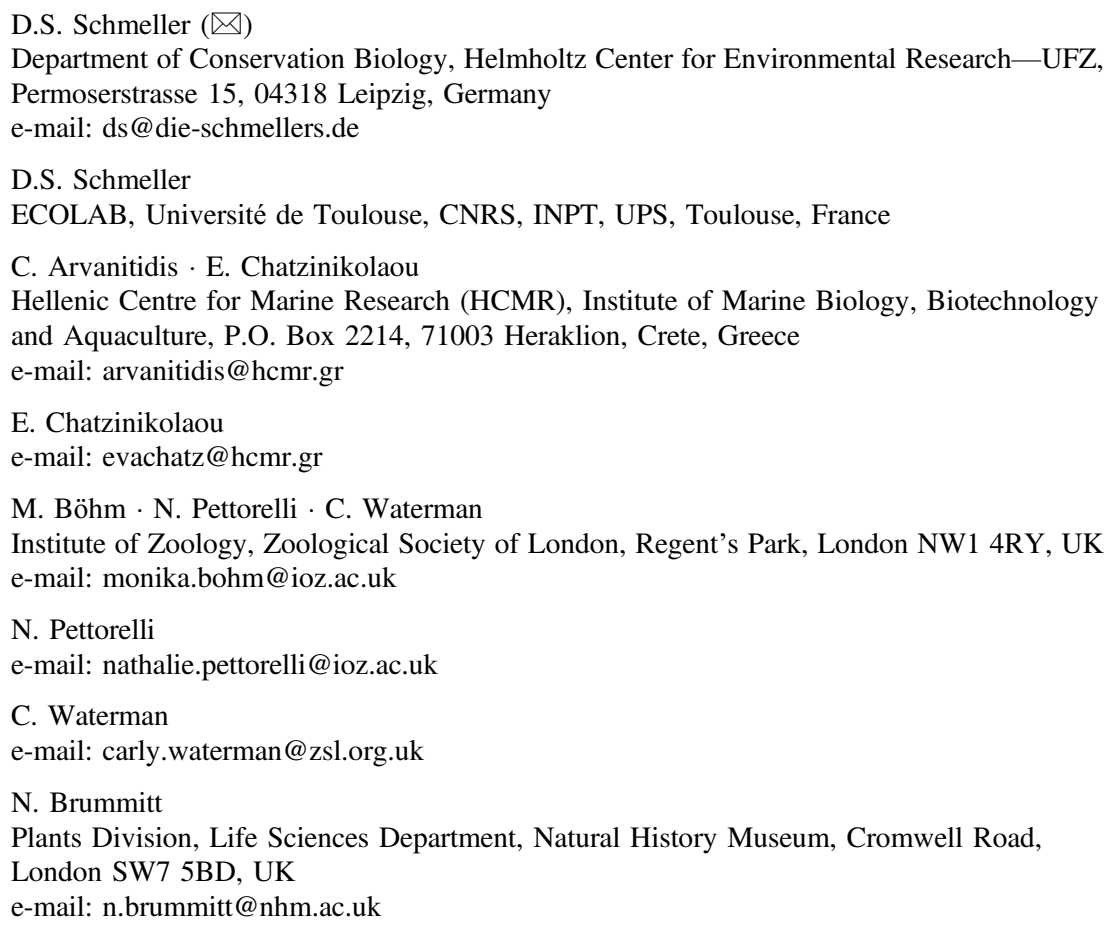


M.J. Costello

Faculty of Science, Marine Science, Leigh Science Building, 160 Goat Island Rd, Leigh 0985, New Zealand

e-mail: m.costello@auckland.ac.nz

H. Ding $\cdot$ C. Peng $\cdot$ J. Wu $\cdot$ H. Xu

Nanjing Institute of Environmental Sciences, Ministry of Environmental Protection, Nanjing 210042, People's Republic of China

e-mail: nldinghui@qq.com

C. Peng

e-mail: cuipeng1126@163.com

J. Wu

e-mail:wujun@nies.org

H. Xu

e-mail: xhg@nies.org

M.J. Gill

Environment Canada, 91780 Alaska Highway, Whitehorse, YT Y1A 5B7, Canada

e-mail: mike.gill@polarcom.gc.ca

P. Haase · J.P. Simaika

Department of River Ecology and Conservation, Research Institute Senckenberg, Clamecystraße 12, 63571 Gelnhausen, Germany

e-mail: peter.haase@senckenberg.de

J.P. Simaika

e-mail: john.simaika@senckenberg.de

P. Haase

Faculty of Biology, Department of River and Floodplain Ecology, University of Duisburg-Essen, Universitãtsstr. 5, D-45141 Essen, Germany

R. Julliard

Muséum National d'Histoire Naturelle, Département Ecologie et Gestion de la Biodiversité, UMR 7204 MNHN-CNRS Centre d'Ecologie et des Sciences de la Conservation, 55 rue

Buffon, 75005 Paris, France

e-mail: julliard@mnhn.fr

J. García-Moreno

ESiLi, Het Haam 16, 6846 KW Arnhem, The Netherlands

e-mail: jaime_gm@yahoo.com

U. Schmiedel

Biocentre Klein Flottbek and Botanical Garden, University of Hamburg, Ohnhorststrasse 18, 22609 Hamburg, Germany

e-mail: ute.schmiedel@uni-hamburg.de

C. Riginos

Teton Science School, 700 Coyote Canyon Road, Jackson Hole, WY 83001, USA

e-mail: Corinna.Riginos@tetonscience.org

J. Belnap

U.S. Geological Survey, Southwest Biological Science Center, Moab, UT 84532, USA

e-mail: jayne_belnap@usgs.gov 
efforts to illustrate how capacity building efforts are being conducted at different geographic scales and under a range of resource, literacy, and training constraints. Accordingly, we include examples of monitoring efforts from within countries (Kenya, France, and China), within regions (Central America and the Arctic) and larger capacity building programs including EDGE (Evolutionarily Distinct and Globally Endangered) of Existence and the National Red List Alliance.

Keywords Monitoring capacity building - Citizen science and volunteers - Key biodiversity areas $\cdot$ Public awareness raising

\subsection{Introduction}

Monitoring the status and trends of species is critical to their conservation and management. However, the current state of biodiversity monitoring is insufficient to detect such for most species and habitats, other than in a few localised areas. One of the biggest obstacles to adequate monitoring is the lack of local capacity to carry out such programs. Thus, building the capacity to do such monitoring is imperative. The capacity building needed includes finding stable lead institutions with adequate funding and staff, and the training of local personnel in the development of new programs of biodiversity monitoring where gaps currently exist and linking together existing and planned observation systems around the world (Henry et al. 2008). In addition, common technical standards among all monitoring efforts are needed, such that data from the huge variety of national monitoring programs, regional biodiversity observation networks (RBONs), and global non-governmental organisations (NGOs) can be combined into coherent data sets that allow the assessment of status and trends of biodiversity across the world (Hoffmann et al. 2014). However, it is clearly unrealistic to attempt simultaneous monitoring of all species in all places. Therefore, the first step is to identify and focus on topical priorities for a given monitoring effort (e.g., species or habitats of special concern) to determine where to focus initial programs. These efforts can then be subsequently complemented by other local, national and regional monitoring activities determined by national responsibilities (e.g., Schmeller et al. 2008a, b, 2012) or topical priorities (Henle et al. 2013).

In this chapter we highlight different biodiversity monitoring efforts to illustrate how capacity building efforts are being conducted at different geographic scales and under a range of resource, literacy, and training constraints. Accordingly, we include examples of monitoring efforts from within countries (Kenya, France, and China), within regions (Central America and the Arctic) and larger capacity building programs including EDGE (Evolutionarily Distinct and Globally Endangered) of Existence and the National Red List Alliance, which are capacity building frameworks similar in structure to Group on Earth Observations-Biodiversity Observation Network (GEO BON). 


\subsection{Building Monitoring Capacity at the Country Scale}

\subsubsection{Kenya}

Northern Kenya is a region of high biodiversity conservation value. It is a hotspot of mammalian diversity in Africa and is critical habitat for several endangered and threatened large mammal species. This region is largely under tribal communal tenure, and the rich flora and fauna of the region are threatened by heavy and continuous grazing by domestic livestock, which has caused moderate to severe land degradation over large areas (Georgiadis et al. 2007).

Several NGOs, the largest of which is the Northern Rangelands Trust (NRT), have been working to promote wildlife conservation and better land stewardship in this region. In 2008, NRT and other scientists developed a simple protocol useable by community members to monitor rangeland condition, which, in turn, determines the amount and quality of forage for wildlife. These methods were based upon pastoralists' traditional knowledge and monitoring practices that were also be scientifically defensible. Through a series of conversations at which the NGOs, scientists, and community members were present, all participants agreed that the indicators currently used by pastoralists to make management decisions were insufficient, as they were largely focused on livestock condition and grass conditions affected by rainfall. All agreed that a new protocol was needed to capture information about long-term ( $>3$ year) trends in rangeland functionality. However, these methods would need to be simple so that they could be learned and used by community members with low literacy.

With support from the United States Agency for International Development (USAID-East Africa), a team of scientific experts was assembled, as well as a diverse advisory panel, to guide this project. This team conducted focus group interviews with community members representing the Maasai, Samburu, Borana, Afar, and Karyu ethnic groups in Kenya and Ethiopia. In these interviews, researchers asked community members to describe the changes in their rangelands they had observed over the last several decades. The responses were markedly similar across diverse social and ecological contexts: increased bare ground, decreased perennial grasses, increased woody vegetation, and increased soil erosion. These observations also matched the general indicators of degradation that have been observed by scientists working in rangelands around the world.

Researchers then used this traditional knowledge to adapt an existing set of rangeland monitoring methods (originally developed and thoroughly tested in the western United States) for the East African context. Notably, the US methods were simplified by basing them on a stick $1 \mathrm{~m}$ long (rather than measuring tapes or other manufactured tools) and created a single graphical data sheet on which data could be collected by circling icons. The intent was that these methods could be used by people who could not read or write but could count and recognise simple icons (e.g., a picture of a shrub representing a sample point with shrub cover). 
At the same time, it was agreed with NRT and other partners that a person with a higher level of education would assist with the design for data collection (e.g., selecting sites and deciding upon number of replicates), training of the pastoralists, and the analysis and interpretation of results. To guide this process, a 50+ page manual was developed to explain the core methods, as well as the key steps to designing, implementing, and describing results from a monitoring program to the communities. This guidebook included numerous photos and graphics to illustrate messages in an accessible format.

The first version of the data collection protocol and the guide book were then subject to rigorous and critical feedback from a diverse community of development and conservation practitioners, community members, and scientists through written feedback, a round-table discussion, and field testing of the methods. Following this process, the data sheets were modified to eliminate some areas of confusion. The guidebook was expanded to a 100 page document with additional case studies and appendices. In 2010, Version II of Monitoring Rangeland Health: A Guide for Pastoralist Communities and Other Land Managers in Eastern Africa was printed in full colour on plastic coated paper to provide a durable and appealing product.

Following release of the printed product, several training sessions were held for the staff of NRT and other key partner organisations. These 'train the trainer' sessions helped to further identify areas of confusion and ensure that the senior staff members were competent in the core field methods (Fig. 13.1).

Guidance was also provided to NRT senior staff as they decided upon a design for their monitoring program. They were then accompanied to the field when they introduced the new monitoring methods in the initial five communities. The NRT

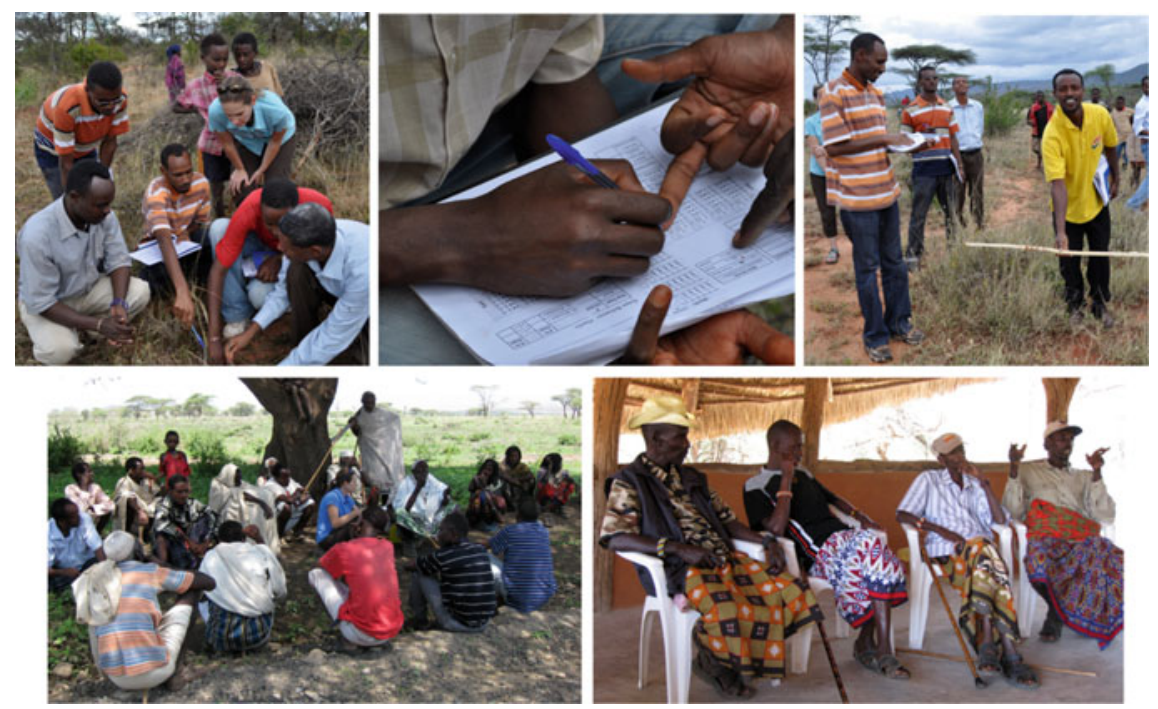

Fig. 13.1 Training sessions of the Northern Rangelands Trust in Kenya. Source Jayne Belnap 
staff trained and supervised community members to collect data, while members of the development team were present to answer any additional questions and observe issues that arose as these methods were implemented in diverse field conditions.

After one year of using these methods, senior NRT staff returned to the development team to discuss possible further simplifications to the core data collection protocol. In their experience, certain data were confusing to collect and certain other data were not necessary to answer the management questions set out by community managers. Some of the simplifications requested were easy to agree upon based on their objectives, while others were considered oversimplifications by the scientific team. Through an extended conversation, new, streamlined protocols that all parties could agree upon were developed and the data sheets modified accordingly (Fig. 13.2).

The NRT staff were very pleased with this product as a data collection tool that could be used by community members, but remained concerned that outside "experts" would be required to analyse and interpret long-term trends, decreasing the likelihood that these results would feed back into community decision-making. They therefore asked for assistance in developing a simple Access database toolwith the objective that a literate community member could enter the data into the database and generate simple graphical reports (e.g., trends in key indicators, such as perennial grass cover, over time and over sites). The development team has since been working with NRT staff and a hired database developer to create this tool.
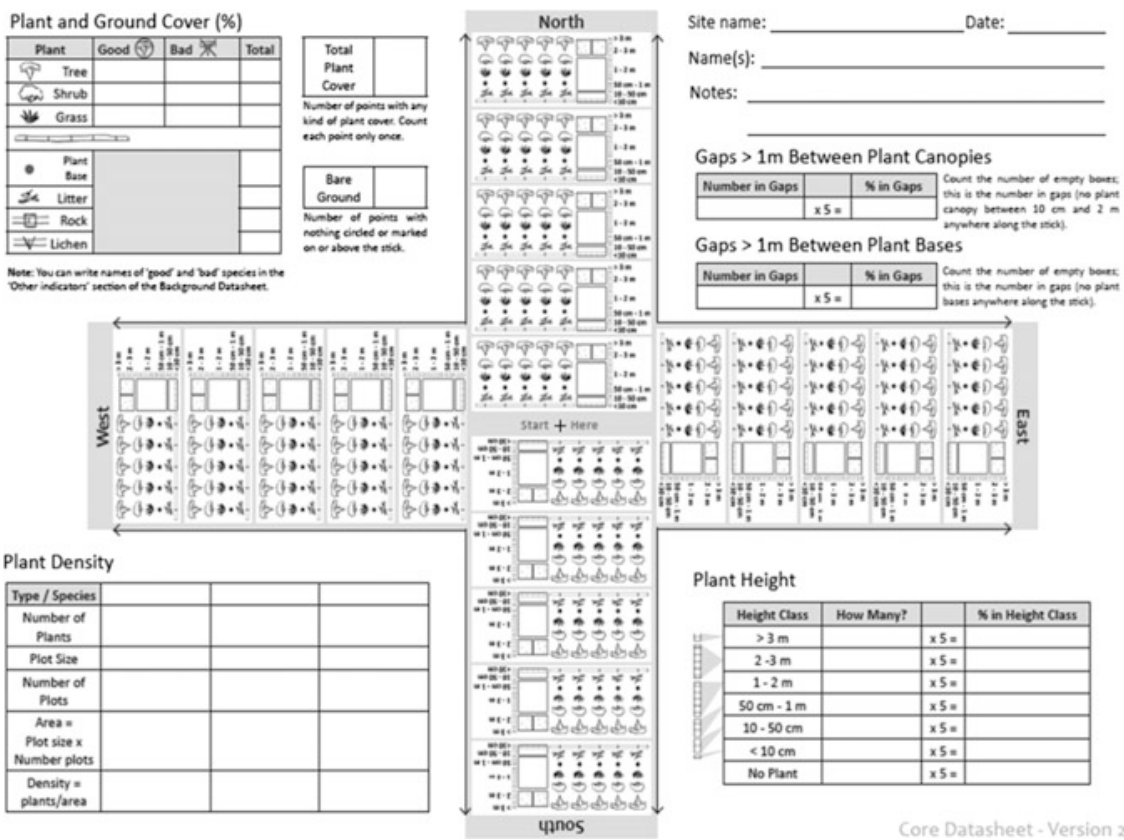

Fig. 13.2 Data collection sheet developed by the Northern Rangelands Trust in Kenya. Source Jayne Belnap 


\subsubsection{France}

To a large extent, biodiversity monitoring in France depends on Citizen Science and thus its success depends on the efficacy of project implementation at a national scale. France has many small national NGOs (e.g., French Bird Life group Ligue de Protection des Oiseaux with about 40,000 members), and these NGOs alone were not able to launch an ambitious national monitoring initiatives. Thus, success of the national effort required a larger institution that could lead and coordinate these smaller efforts. Fortunately, the French National Museum of Natural History (MNHN) was and is able to perform this role. In addition, this research institution is under Ministries who are officially designated role to document the state of biodiversity in France. Therefore, the success of the national monitoring effort in France required both building capacity among the smaller NGOs, as well as a government institution with stable funding and staff to provide a common monitoring framework.

Integrating the individual Citizen Science-driven monitoring programs started with MNHN hosting the bird ringing project, a very successful a partnership between professional researchers and volunteers. The MNHN also launched a classical Breeding Bird Survey (BBS), again using the Citizen Science provided by the smaller NGOs [together, these formed the STOC (Le Suivi Temporel des Oiseaux Communs - Vigie Nature $=$ Temporal Survey of Common Birds) program]. Several factors facilitated the success of these schemes: (1) coupling the BBS to the already-existing ringing efforts; (2) focusing on common birds, thus avoiding an overlap with other projects concerned with all species (and thus proportionally more rare species); (3) the concomitant emergence of biodiversity indicator based monitoring schemes, based on Mean Species Abundance, (e.g., the Breeding Bird Survey), ensuring considerable political interest in these schemes, and (4) this effort coincided with citizen science becoming fashionable in France.

With the success of the national bird monitoring effort, two new schemes were put in place to develop capacity for monitoring other taxa. The first was based on the same logic as the Breeding Bird Survey: training and motivating skilled amateurs to collect data following a protocol and a sampling design for butterflies, bats plants, and dragonflies. The second program was developed to train and coordinate efforts of the general public to monitor garden butterflies and snails, bumblebees, birds, flower-dwelling insects, and wild plants in cities. All these schemes are coordinated by the same scientific team based at the MNHN, but each also relies on a specific NGO partner, which is dedicated to the success of the (specific) participant network. The NGO partner trains participants, ensures that each new scheme capitalises directly on preceding experience to maximise the chance for joint data analysis, and coordinates all efforts with MNHN. Several characteristics have made this effort an outstanding success: (1) different monitoring schemes for different species groups were integrated from the beginning, (2) the same research group was involved in citizen science schemes for both skilled amateurs and the general public, (3) strong involvement of researchers in designing citizen science projects 
maximised the chances that the future database will allow sophisticated and robust statistical analyses of the large datasets and (4) development and training of personnel in the use of technology to facilitate reliable data where detection or identification is difficult (e.g., ultrasound recording for bats, photography for spiders and flower-dwelling insects). Combined, such an organisation is very cost-effective, as researchers are keen to commit themselves to such projects to ensure good quality data.

Three additional planned projects will expand the span of national biodiversity monitoring through citizen science. The first is to implement more experimental approaches in addition to simple counts. This opens scientific opportunities while keeping participants motivated to participate by offering renewed ways of looking at biodiversity. The second project expands citizen science to primary and secondary schools. Together with educational staff, students will collect 'real' data (i.e., using the same protocol as the general public) during school time (i.e., as part of the official school program). The ultimate target is for half of French children to experience citizen science at least once during their schooling. A third project is to work with farmers, encouraging them to monitor biodiversity on their farms, an approach that worked well with 400 farmers in the first year of its existence. Working with local farmer organisations was essential in this effort, but the combined launched of this project with the MNHM guaranteed its integrity and longevity.

\subsubsection{China}

The first ecological research station was developed in China in 1978. In 1988, the China Ecosystem Research Network (CERN) was established by the Chinese Academy of Sciences. It includes 39 research stations that include ecosystems as diverse as farmland, forest, grassland, desert, marsh, lake, ocean, and cities. At each station, the structure, function and dynamic patterns of the ecosystem, as well as abiotic measures, is recorded. In 2003, the China Forestry Ecosystem Research Network (CFERN) was established, followed by the China Wetland Ecosystem Research Network (CWERN) and the China Desert Ecosystem Research Network (CDERN). In 2005, CFERN, CWERN, and CDERN were combined to form the China National Ecosystem Research Network (CNERN). In addition to CNERN, the Ministry of Agriculture also launched the China National Grassland Resource Monitoring project (CNGRM) in 2005. CNGRM focuses on the monitoring of vegetation growth, productivity, and utilisation, as well as the effects of disaster conditions and construction projects on biodiversity. Planning, establishing, and running such a large network of sites has required stable institutions and funding to build the needed scientific capacity to organise and guide this effort, as well as to recruit and train volunteers to help with data collection. For example, in 2012, over 4500 volunteers and professionals were trained and organised to measure 8000 plots in over 450 counties in 23 Chinese provinces. Most of the field workers were 
technical staff of local administrations, complemented by students from agricultural universities.

While ecosystem level monitoring has been on-going for some time, species level monitoring networks were established in 2005. These have included a coastal waterbird project, covering nearly all the wetland sites along coastal areas of the East and South China Sea (Fig. 13.3), utilizing about 150 volunteers. In 2011, the Nanjing Institute of Environmental Sciences, under the umbrella of the Ministry of Environmental Protection of China, began a bird and amphibian monitoring effort. Again, this project was planned, developed, and coordinated by scientists, but the field surveys were conducted by workers from colleges, research institutes, museums, and other organisations that were recruited and trained by the scientists.

Despite these efforts, more biodiversity monitoring is needed at the national level, covering major ecosystems and indicator species, to better understand the status and trends of biodiversity in China. In 2014, the Ministry of Environmental Protection of China began developing a biodiversity monitoring network. Based on the existing organisations and frameworks, this effort will start with further development of a bird and amphibian monitoring network (Fig. 13.3). However, the end goal is a comprehensive national monitoring scheme that covers mammals, birds, reptiles, amphibians, fishes, and vascular plants, with a special focus on endangered species. Thus, similar to France, successful monitoring efforts in China

(a)

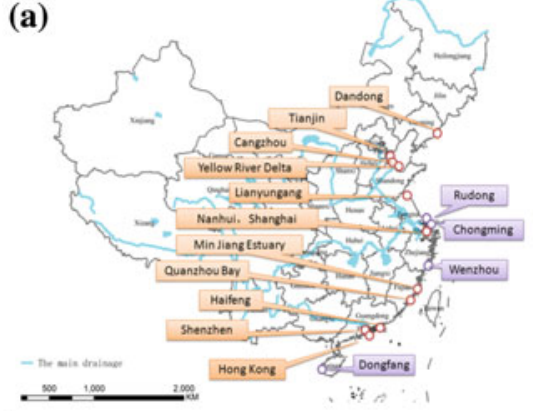

(c)

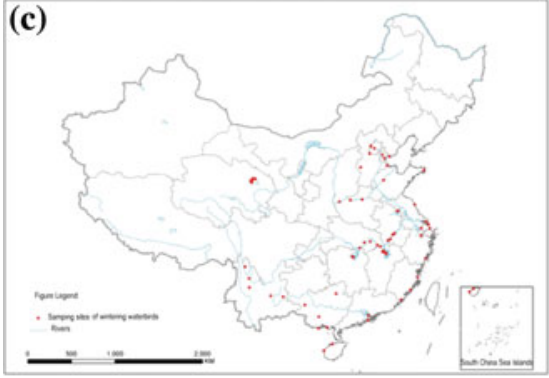

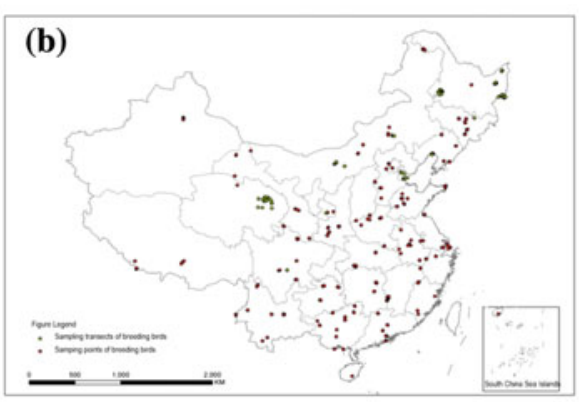

(d)

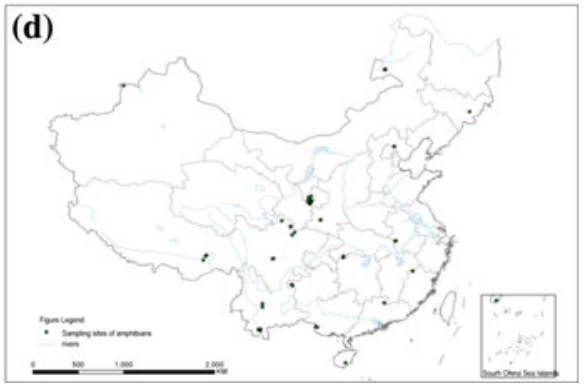

Fig. 13.3 Distribution of biodiversity monitoring sites in China for a the coastal wetland monitoring scheme of China, $\mathbf{b}$ the breeding bird survey of China, $\mathbf{c}$ the wintering water bird survey of China, and $\mathbf{d}$ the amphibian monitoring sites of China. Source Cui Peng 
have relied on a stable source of funding (e.g., the government) and the scientific expertise needed to plan, develop and execute successful monitoring protocols, as well as to recruit and train volunteers for data collection.

\subsection{Building Monitoring Capacity at the Regional to National Scale}

\subsubsection{Pan-Arctic}

Arctic ecosystems and the biodiversity they support are experiencing growing pressure from various stressors (e.g., development, climate change, contaminants). However, established research and monitoring programs have remained largely uncoordinated, and therefore lack the ability to effectively monitor, understand and report on biodiversity trends at the pan-Arctic or regional scale (MA 2005). The maintenance of healthy arctic ecosystems is a global imperative, as the Arctic plays a critical role in the Earth's physical, chemical and biological balance. A coordinated and comprehensive effort for monitoring Arctic ecosystems is needed to facilitate effective and timely conservation and adaptation actions.

While all Arctic states, as well as a number of non-Arctic states and organisations, conduct monitoring of various elements of Arctic biodiversity, the lack of coordination has limited their geographic, thematic, and temporal scope and are not evenly spread across the Arctic. In particular, northern areas of Canada, Greenland and Russia have very limited biodiversity monitoring, whereas areas in northern Scandinavia, the Bering Sea, Aleutian Islands and Iceland have relatively intense, on-going biodiversity monitoring (Fig. 13.4) and in many cases, long-term datasets. Given that the area in question is 32 million $\mathrm{km}^{2}$ (three times the size of Europe) and is comprised of largely remote and extreme ecosystems, it is not surprising that current biodiversity monitoring efforts are seen as inadequate. Indeed, recent issues regarding state finances and priorities have made it more difficult to sustain even existing efforts.

The current situation facing the Arctic demands a well-designed, scaled, pan-arctic, ecosystem-based approach that not only identifies trends in biodiversity, but also identifies underlying causes of these trends. It is critical that this information be made available, as plans for adaptation and mitigation need development, which ultimately depend on rigorous, integrated and efficient monitoring programs that have the power to detect change within a 'management' time frame.

To meet these challenges, the Conservation of Arctic Flora and Fauna (CAFF) Working Group of the Arctic Council launched the Circumpolar Biodiversity Monitoring Program (CBMP) in 2005. The CBMP is working with over 80 global partners in building the capacity to expand, integrate and enhance existing Arctic biodiversity monitoring efforts, thus facilitating more rapid detection, communication and response to significant trends and pressures. It is strategically linked to a 


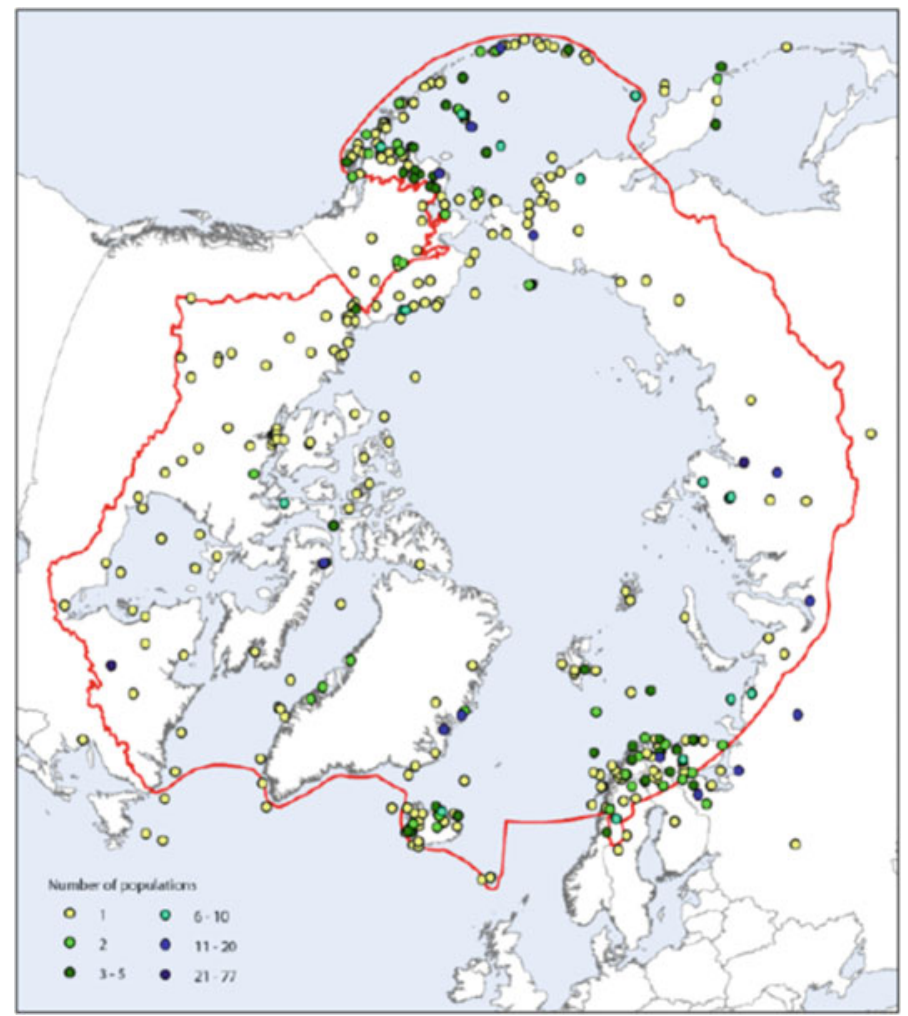

Fig. 13.4 Distribution of population time series data across the Arctic, 1951-2010. The number of populations per location is indicated by colour. The red line indicates the core area of the Circumpolar Biodiversity Monitoring Program. Source Arctic climate impact assessment (2005)

number of international conventions and programs including the Convention on Biological Diversity (CBD), UNEP's Biodiversity Indicators Partnership and is one of four recognised regional Biodiversity Observation Networks of the GEO BON initiative. In order to connect the diversity of biodiversity observing networks, such as scientific field stations, community-based monitoring programs, theme-based monitoring networks (e.g., caribou) operating at different scales across the Arctic, the CBMP is establishing four Expert Monitoring Groups representing major Arctic themes (Marine, Freshwater, Terrestrial and Coastal). Each group, representing a diversity of disciplines, is tasked with developing and implementing pan-arctic integrated biodiversity monitoring plans with a focus on harmonizing existing monitoring networks and methodologies, as well as rescuing and aggregating existing data to establish historical baselines. To date three (Marine, Freshwater and Terrestrial) monitoring plans have been developed and are being implemented. To facilitate effective reporting and data management, the CBMP has developed a number of headline indicators targeting CBD 2020 Targets (McRae et al. 2012) as 
well as a web-based data portal (Arctic Biodiversity Data Service www.abds.is; http://www.youtube.com/watch? $\mathrm{v}=\mathrm{ONdmmIcuqNE}$ ) that is improving metadata and the discovery, access, and interpretation of data to bridge the science-policy gap. The output from the CBMP biodiversity monitoring plans is being used to populate both the ABDS and headline indicators which, in turn, are being translated into policy-targeted reports sub-national, national and regional (e.g., annual Arctic Report Cards http://www.arctic.noaa.gov/reportcard/index.html) in scope to facilitate more timely and effective decision-making.

The CBMP's approach with regard to developing the pan-Arctic monitoring plans is to first identify the sub-national, national, and regional reporting mandates of governments relevant to biodiversity. Implementation of the monitoring plans will only be sustained if they can provide information that supports these mandates. The next steps are to (1) develop conceptual models of the ecosystems/biomes in question and through an iterative process, (2) identify the priority focal ecosystem components (FECs) and processes that should be monitored, (3) identify the biodiversity variables (e.g., attributes) and specific parameters for these FECs that should be measured, and (4) identify common methodological approaches and sampling frameworks for measuring these parameters. In most cases, these monitoring plans focus on ways to harmonise existing methodologies and data, rather than standardise them, as many monitoring networks have been using particular methodologies and data standards for many years and are unlikely to change their approach. Where new variables are proposed, an opportunity to adopt a specific methodology and standard is available and recommendations are made.

The implementation of these monitoring plans will then involve building national or thematic teams that allows for a hierarchical and efficient approach to connect to the many monitoring networks and practitioners operating at different scales across the Arctic. The resulting data will be mostly managed within existing national biodiversity data centres which the ABDS can access via the Internet. This will provide an efficient means to access the most up to date information on various aspects of Arctic biodiversity status and trends. The development of the CBMP grew from a concept team of ten in 2005 to over 80 organisations representing hundreds of scientists and local resource users around the Arctic in 2013 with a concurrent ten-fold increase in its budget over this time-span.

\subsubsection{Central America}

Key Biodiversity Areas (KBAs) are sites of global importance for conservation of biodiversity. As an important tool in conservation planning, these areas are considered critical for the persistence of one or more globally threatened species and are identified using simple standardised occurrence data (Eken et al. 2004). This approach has been modified to identify important sites for different taxonomic groups, such as important bird areas (developed by BirdLife-www.birdlife.org); plant areas (Anderson 2002), butterfly areas (van Swaay and Warren 2003), or for 
specific biomes (e.g., freshwater key biodiversity areas; Darwall et al. 2011). This is an international effort currently led by the International Union for the Conservation of Nature (IUCN).

This same process is also being used at the national scale. In Central America, the NGO Conservation International identified a local partner institution in each of the seven countries and built up their capacity to identify KBAs in each country. This required gathering occurrence data for species assessed as Vulnerable, Endangered, or Critically Endangered by the IUCN Red List, using as many sources as possible globally. This, in turn, provided each country with a more complete set of records for species of interest. With the help of Conservation International, each institution involved then defined the KBAs for these species, while also defining monitoring objectives. All collected information is entered into a global database - the World Biodiversity Data Base, currently managed by BirdLife International - and shared with the relevant authorities of the different countries and regional bodies (e.g., Central American Commission for the Environment and Development).

\subsection{Building Monitoring Capacity at the National and Global Scales}

\subsubsection{International Union for the Conservation of Nature Programs}

The IUCN Red List of Threatened Species is arguably one of the most important tools for global nature conservation. It facilitates the flow of biodiversity information from the point of data collection to policy- and decision-makers around the world, and drives research into biodiversity conservation. However, such lists at the national scale are also needed and this will require capacity building within each country. The production of National Red Lists is gaining momentum (Miller et al. 2007; Zamin et al. 2010), as they provide a valuable tool for national decision-making and priority-setting for conservation, while also aiding national reporting against global biodiversity targets such as the Aichi Biodiversity Targets (Szabo et al. 2012). Integrating global and national Red List processes provides one way in which we can dramatically increase taxonomic and geographic coverage of the IUCN Red List (Rodriguez 2008), while national assessments can benefit greatly from the expertise provided by the IUCN Red List in conducting species assessments and utilising the data for maximum conservation benefit, thus building capacity and filling gaps from global to regional scales and vice versa (see also Schmeller et al. 2014).

Previous efforts promoting National Red Lists have primarily focused on the establishment of an online resource for Regional and National Red Lists (see www. nationalredlist.org). The website provides a hub for collating National Red Lists 
and Action Plans from around the world and at present holds in its online library more than 140 National Red Lists and Action Plans from 33 countries. It also contains a species database with more than 85,000 national assessments for over 60,000 species. This has also sparked gap analyses in the global coverage of National Red Lists, which can help in prioritising funding for National Red List development (Zamin et al. 2010).

While this centralisation has provided a good starting point for promoting National Red Lists and Action Plans, information flows much more effectively if regional hubs are established as well, again requiring the recruitment and training of local personnel. Efforts have recently been made to formalise the development of National Red Lists (NRLs) under the auspices of the ZSL and IUCN via the newly created National Red List Alliance (NRLA). The Alliance builds on previous steps taken at international congresses, e.g., the IUCN World Conservation Congress 2012 in Jeju, South Korea, to promote National Red Lists and seek discussion with interested partner organisations. The efforts culminated in a workshop held at the Zoological Society in London in 2013 to formalise the partnership. The aim of the Alliance is to create a regional network of National Red List partners committed to supporting the development and implementation of National Red Lists, with members acting as focal points for National Red Lists. Initial regional hubs will be established in China, Brazil and South Africa, all of which have at present a strong presence in terms of National Red List development. It is hoped that the strengthened network and the joint ownership of the National Red List website will facilitate capacity building where needed as well as ensure the sharing of national-level tools and data for compilation and analysis of National Red Lists, promote the upload of additional species assessments to the National Red List website, and promote sharing of resources for training and best practice for National Red List development.

These programs show that in order to achieve a monitoring programme that is global in coverage but sufficiently resolved spatially to allow national decision making (Scholes et al. 2012), building networks with strong capacity is of utmost importance. Networks function most efficiently via key individuals or institutions, which are inter-linked with each other and which can act as regional hubs to interact with many individuals/institutions at a more local level.

\subsection{The Conservation Leadership Programme and EDGE of Existence Programme}

\subsubsection{The Conservation Leadership Programme}

An important aspect of building capacity is the training of young professionals. The Conservation Leadership Programme (CLP-www.conservationleadership programme.org) is an example of a partnership that has been training 
conservationists for over two decades and now has a broad network of alumni around the world. The Zoological Society of London (ZSL) has also helped establish a number of global networks for the purposes of monitoring and conservation, mostly through training conservationists in wildlife management techniques.

\subsubsection{EDGE of Existence Programme}

The ZSL is also actively working towards conservation of evolutionarily distinct and globally endangered (EDGE) species. As the reason most of the top 100 EDGE species are generally ignored conservation is because they occur in countries where the capacity for effective monitoring and conservation is lacking (primarily developing countries). Hence, to conserve EDGE species, ZSL has developed a grassroots capacity building program that focuses on training and supporting aspiring in-country conservation scientists to establish larger-scale conservation projects in which long-term monitoring of status and threats to EDGE species and their habitats will be a major component. This is accomplished by providing two years funding to study a priority EDGE species, attend regional training courses, study online modules in relevant topics, receive one-to-one support from a scientific advisor based at ZSL or a partner organisation, and at the end, attend a two-week conservation leadership course that includes modules on leadership and management, project planning, monitoring and evaluation, facilitation and conflict resolution, communication skills, proposal writing, and writing for publication, as well as technical one-to-one clinics to help with analysing data and writing up the results of their Fellowship projects (for details on the fellowship, see www. edgeofexistence.org/conservation/become_fellow.php). To date, EDGE has supported 41 EDGE Fellows focusing on 39 EDGE species in 26 countries since 2007, with $97 \%$ of the Fellows still working in conservation and research. These early successes of the EDGE of Existence program suggest that targeted funding and training of key individuals can help to build lasting networks for conservation. The approach taken here is via a structured Fellowship program, although this approach can easily be adapted to target key institutions in key countries to expand the global biodiversity monitoring network.

\subsection{Cross Cutting Lessons from Capacity Building Efforts}

The need to monitor biodiversity is becoming increasingly apparent to scientists, the public, and policy makers around the world. However, because the highest level of biodiversity is located in developing countries where funding, biodiversity 
institutions and formal skills may be limited, capacity building is especially urgent in these regions. Whereas each nation and region has its specific challenges in meeting this need, there are several cross-cutting lessons that can be learned from past and current efforts. These include: Identify a well-known organisation with a stable source of adequate funding and staffing to design and implement the monitoring program. This may be a governmental organisation, a network of NGOs, or other groups. Where possible, this organisation should already have an established network throughout the region.

- Develop and maintain a simple, efficient internal organisational structure with roles of team members clearly defined;

- Include people of influence ('champions') within national governments and funding sources in the program's governance structure;

- Utilise a close partnership among practitioners, NGOs, and scientists in designing and implementing the monitoring program. Identify talented and driven individuals and key institutions to receive training or take part in the monitoring network. Include a mix of young and senior experts in the design and implementation of the program components to ensure program integrity and continuity;

- Develop a very focused and detailed implementation plan that is closely adhered to during the development of the program;

- Focus on harmonizing existing monitoring capacity and information to increase statistical power and cost-efficiencies, rather than attempting to impose new standards on existing programs or developing new monitoring programs;

- Ensure a reasonable allocation of funds for data management, analysis and reporting as well as on communications (program promotion) and fundraising;

- Ensure outputs are relevant to both decision-makers and funders while maintaining scientific integrity, the latter achieved through having engagement with not only scientists and local peoples, but also decision-makers and funders in the program design;

- Heighten public interest and concern to increase funding opportunities;

- Start small and build support for the program through the promotion of early results that showcase the value-added gains for scientists and decision-makers in coordinating and scaling existing biodiversity monitoring efforts;

- Develop a program 'brand' that positions the program as the source for credible information on biodiversity monitoring for the region and one whose endorsement is sought after by other monitoring networks;

- Practice regular communication from conception through design, implementation, testing, and refinement of the monitoring effort. This iterative process is key to building trust between the scientific development team and the intended audience of practitioners;

- Where possible, integrate efforts with an international political body that can provide a more formal mechanism and mandate for engaging scientists, local 
peoples and other monitoring networks and coordinate or take advantage of the publicity associated with other events, such as the International Polar Year of 2007/08;

- Provide continuing support and mentorship to individuals and organisations involved in the monitoring effort, especially in developing countries.

Open Access This chapter is distributed under the terms of the Creative Commons Attribution-Noncommercial 2.5 License (http://creativecommons.org/licenses/by-nc/2.5/) which permits any noncommercial use, distribution, and reproduction in any medium, provided the original author(s) and source are credited.

The images or other third party material in this chapter are included in the work's Creative Commons license, unless indicated otherwise in the credit line; if such material is not included in the work's Creative Commons license and the respective action is not permitted by statutory regulation, users will need to obtain permission from the license holder to duplicate, adapt or reproduce the material.

\section{References}

Anderson, S. (2002). Identifying important plant areas: A site selection manual for europe, and a basis for developing guidelines for other regions of the world. Plantlife International: London.

Arctic climate impact assessment. (2005). Arctic climate impact assessment. Cambridge: Cambridge University Press.

Darwall, W. R., Holland, R. A., Smith, K. G., Allen, D., Brooks, E. G., Katarya, V., et al. (2011). Implications of bias in conservation research and investment for freshwater species. Conservation Letters, 4, 474-482.

Eken, G., Bennun, L., Brooks, T. M., Darwall, W., Fishpool, L. D., Foster, M., et al. (2004). Key biodiversity areas as site conservation targets. BioScience, 54, 1110-1118.

Georgiadis, N., Olwero, J., Ojwang, G., \& Romanach, S. (2007). Savanna herbivore dynamics in a livestock-dominated landscape: I. Dependence on land use, rainfall, density, and time. Biological Conservation, 137, 461-472.

Henle, K., Bauch, B., Auliya, M., Külvik, M., Pe'er, G., Schmeller, D. S., et al. (2013). Priorities for biodiversity monitoring in Europe: A review of supranational policies and a novel scheme for integrative prioritization. Ecological Indicators, 33, 5-18.

Henry, P. Y., Lengyel, S., Nowicki, P., Julliard, R., Clobert, J., Celik, T., et al. (2008). Integrating ongoing biodiversity monitoring: Potential benefits and methods. Biodiversity and Conservation, 17, 3357-3382.

Hoffmann, A., Penner, J., Vohland, K., Cramer, W., Doubleday, R., Henle, K., et al. (2014). The need for a biodiversity policy support process - Building the European contribution to a global Biodiversity Observation Network (EU BON). Nature Conservation, 6, 49-65.

MA (Millennium Ecosystem Assessment). (2005). Ecosystems and human well-being: Synthesis. Washington, DC: Island Press. http://www.millenniumassessment.org/documents/document. 356.aspx.pdf

McRae, L., Böhm, M., Deinet, S., Gill, M., \& Collen, B. (2012). The Arctic Species Trend Index: Using vertebrate population trends to monitor the health of a rapidly changing ecosystem. Biodiversity, 13, 144-156.

Miller, R. M., Rodriguez, J. P., Aniskowicz-Fowler, T., Bambaradeniya, C., Boles, R., Eaton, M. A., et al. (2007). National threatened species listing based on IUCN criteria and regional guidelines: Current status and future perspectives. Conservation Biology, 21, 684-696. 
Rodriguez, J. P. (2008). National Red Lists: The largest global market for IUCN Red List categories and criteria. Endangered Species Research, 6, 193-198.

Schmeller, D. S., Gruber, B., Bauch, B., Lanno, K., Budrys, E., Babij, V., et al. (2008a). Determination of national conservation responsibilities for species conservation in regions with multiple political jurisdictions. Biodiversity and Conservation, 17, 3607-3622.

Schmeller, D. S., Gruber, B., Budrys, E., Framsted, E., Lengyel, S., \& Henle, K. (2008b). National responsibilities in European species conservation: A methodological review. Conservation Biology, 22, 593-601.

Schmeller, D. S., Maier, A., Bauch, B., Evans, D., \& Henle, K. (2012). National responsibilities for conserving habitats-A freely scalable method. Nature Conservation, 3, 21-44.

Schmeller, D. S., Evans, D., Lin, Y. P., \& Henle, K. (2014). The national responsibility approach to setting conservation priorities-Recommendations for its use. Journal for Nature Conservation, 22, 349-357.

Scholes, R. J., Walters, M., Turak, E., Saarenmaa, H., Heip, C. H., Tuama, E., et al. (2012). Building a global observing system for biodiversity. Current Opinion in Environmental Sustainability, 4, 139-146.

Szabo, J. K., Butchart, S. H. M., Possingham, H. P., \& Garnett, S. T. (2012). Adapting global biodiversity indicators to the national scale: A Red List Index for Australian birds. Biological Conservation, 148, 61-68.

van Swaay, C., \& Warren, M. (2003). Prime butterfly areas in Europe: Priority sites for conservation. Ministry of Agriculture, Nature Management and Fisheries.

Zamin, T. J., Baillie, J. E., Miller, R. M., Rodriguez, J. P., Ardid, A., \& Collen, B. (2010). National red listing beyond the 2010 target. Conservation Biology, 24, 1012-1020. 